\title{
Methodological Problems in fMRI Studies on Acupuncture: A Critical Review with Special Emphasis on Visual and Auditory Cortex Activations
}

\author{
Florian Beissner ${ }^{1,2,3}$ and Christian Henke ${ }^{1,4}$ \\ ${ }^{1}$ Brain Imaging Center, Goethe-University, Frankfurt, Neuroradiology, Schleusenweg 2-16, 60528 Frankfurt, Germany \\ ${ }^{2}$ Institute of Neuroradiology, Goethe-University, Frankfurt, Germany \\ ${ }^{3}$ Max-Planck-Institute of Biophysics, Goethe-University, Frankfurt, Germany \\ ${ }^{4}$ Department of Neurology, Goethe-University, Frankfurt, Germany
}

Correspondence should be addressed to Florian Beissner, florian.beissner@uni-jena.de

Received 17 June 2009; Accepted 30 August 2009

Copyright (๑) 2011 F. Beissner and C. Henke. This is an open access article distributed under the Creative Commons Attribution License, which permits unrestricted use, distribution, and reproduction in any medium, provided the original work is properly cited.

Functional magnetic resonance imaging (fMRI) has been used for more than a decade to investigate possible supraspinal mechanisms of acupuncture stimulation. More than 60 studies and several review articles have been published on the topic. However, till now some acupuncture-fMRI studies have not adopted all methodological standards applied to most other fMRI studies. In this critical review, we comment on some of the problems including the choice of baseline, interpretation of deactivations, attention control and implications of different group statistics. We illustrate the possible impact of these problems by focussing on some early findings, namely activations of visual and auditory cortical areas, when acupoints were stimulated that are believed to have a therapeutic effect on vision or hearing in traditional Chinese medicine. While we are far from questioning the validity of using fMRI for the study of acupuncture effects, we think that activations reported by some of these studies were probably not a direct result of acupuncture stimulation but rather attributable to one or more of the methodological problems covered here. Finally, we try to offer solutions for these problems where possible.

\section{Introduction}

Since the late 1990s, functional magnetic resonance imaging (fMRI) has been used to investigate the underlying mechanisms of the Chinese medical therapy of acupuncture. After the proposal of the idea [1] the groups of Hui and Cho were the first to show cortical activations associated with acupuncture stimulation using fMRI $[2,3]$. The study by Cho et al. [4] is still referenced in the literature as a breakthrough in acupuncture research. However, this paper was later retracted by five of the eight authors. In the meantime, more than 60 studies have been published that tried to elucidate the underlying mechanisms of acupuncture by means of fMRI. However, researchers working in this field are still facing a number of unsolved problems and up to now some acupuncture-fMRI studies have not adopted all methodological standards applied to most other fMRI studies (Figure 1). In this critical review we would like to comment on some of these problems and try to offer solutions, where possible. We have illustrated the possible impact of these problems by focussing on some early findings that have sparked a lot of interest, namely activations of visual and auditory cortical areas under stimulation of acupoints that are believed to have a therapeutic effect on vision or hearing from the viewpoint of traditional Chinese medicine (TCM). We will show that, there exist several mechanisms that can cause such activations independent of the actual acupuncture stimulation. We will not try to review acupuncture-fMRI studies as a whole since there are excellent and up-to-date reviews available [5-7]. Neither will we cover general problems of acupuncture research like the question of placebo and sham interventions [8] or differences between stimulation methods [9].

\section{Identification of Relevant Literature}

Relevant publications for this review were identified by a systematic search of PubMed using the terms "acupuncture" 


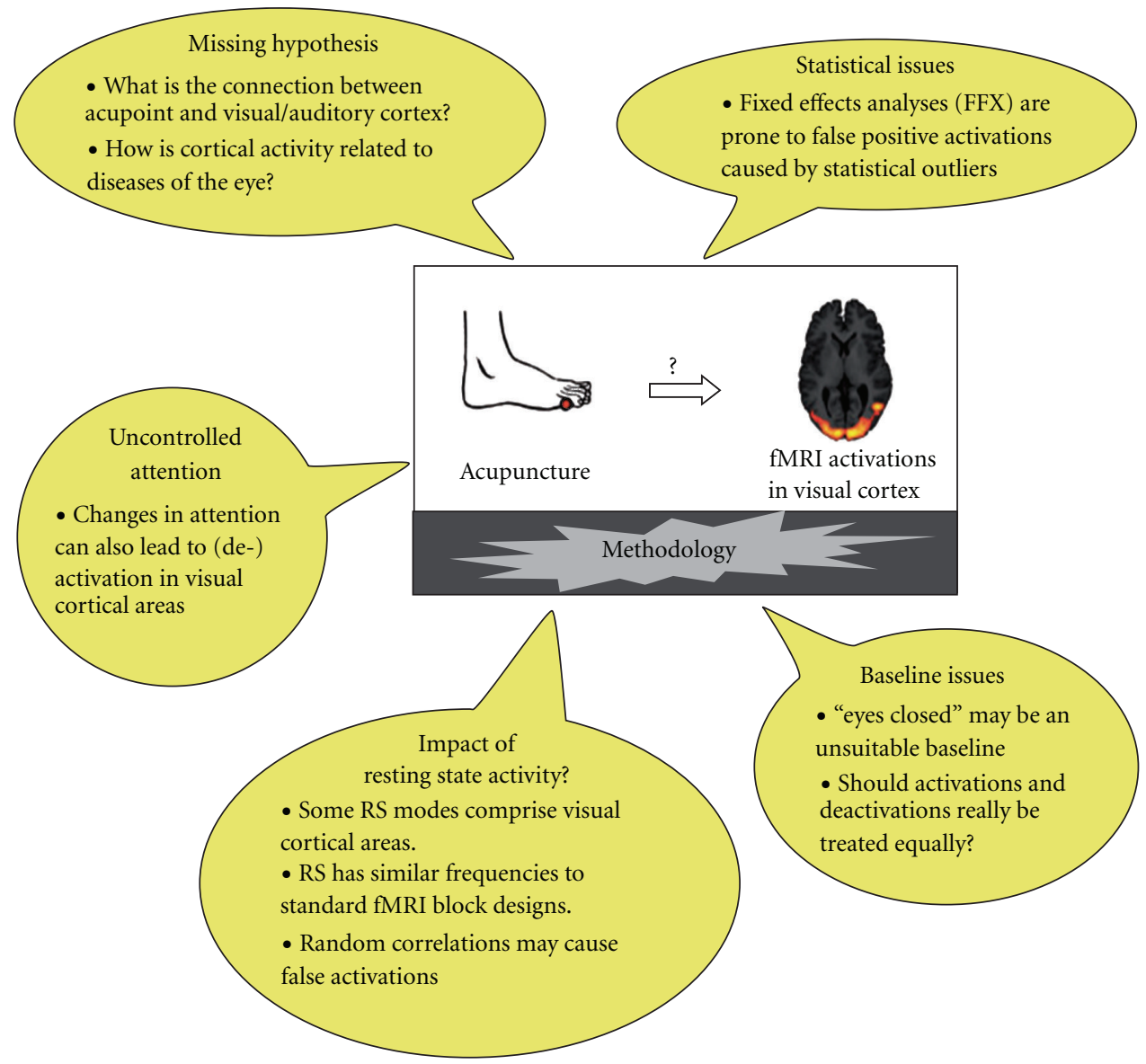

FIGURE 1: Possible explanations other than acupuncture for activations of visual cortical areas found in acupuncture-fMRI studies. Only visual areas have been covered here, although the same reasoning applies to auditory cortical areas.

and "fMRI" and of reference lists of retrieved articles. After discarding unrelated publications we checked all remaining 67 publications (see supplementary material for a complete list) for their choice of acupoints and reported cortical activations. We identified eight studies that investigated the cortical effect of vision-related acupoints [3, 10-16] and two studies that did the same for hearing-related acupoints $[14,17]$. Table 1 gives an overview of all nine studies covered in this review.

\section{General Observations}

3.1. A Comment on Possible Underlying Mechanisms. The putatively logical relationship between the therapeutic potential of vision- or hearing-related acupoints and activations of cortical areas that process these sensations may be one reason for the popularity of the results. However, an anatomical link between the lateral aspect of the foot (acupoints of the BL conduit) and the visual cortex could not be demonstrated so far. The same holds true for the auditory points used. However, such a relationship may exist on the cortical level. As several studies have shown, sensory stimulation can activate both visual and auditory cortices [18-20]. The strong sensory component of acupuncture is self-evident. However, its relation to the underlying mechanisms of acupuncture is yet to be proven. Furthermore, if we take a standard acupuncture book [21] and look up common indications for the two most often used points BL60 and BL67, we mainly find that the eyerelated symptoms ("redness, pain and swelling of the eyes", "eye pain", "pain of the inner canthus", etc.) have nothing to do with the visual cortex. Other indications such as "visual dizziness" and "superficial visual obstruction" also have little or no connection to the function of the visual cortex. Similar arguments apply to auditory acupoints also. Thus, if there is a measurable effect of these acupoints on vision or hearing, it seems unlikely that this effect will take part in the respective cortices.

3.2. Heterogeneity of the Results. Table 1 reveals that the methodology and results of the studies appear quite heterogeneous. All but one study using vision-related acupoints indeed reported to have found activations, deactivations or both in some part of the visual cortex (Brodman areas 17, 18 and 19) [10]. For hearing-related acupoints the situation is similar, although there is only one positive and one negative result here. Cho et al. [22, 23] also published positive results on hearing-related acupoints in two books 
TABLE 1: Methodological and statistical details of acupuncture-fMRI studies reporting activations of visual or auditory cortices under stimulation of respective acupoints.

\begin{tabular}{|c|c|c|c|c|c|c|c|c|c|}
\hline & $n$ & $\begin{array}{l}\text { Block } \\
\text { length }\end{array}$ & $\begin{array}{c}\text { Group } \\
\text { statistics }\end{array}$ & $\begin{array}{c}\text { Correction } \\
\text { method }\end{array}$ & $P$-value & $\begin{array}{c}\text { Stimulation } \\
\text { method }\end{array}$ & $\begin{array}{c}\text { Activations/ } \\
\text { deactivations }\end{array}$ & $\begin{array}{l}\text { (De-)activated } \\
\text { Brodman areas }\end{array}$ & $\begin{array}{c}\text { Acupoints } \\
\text { used }\end{array}$ \\
\hline \multicolumn{10}{|l|}{ Vision-related acupoints } \\
\hline Cho et al. [3] & 12 & $50 \mathrm{~s}$ & None & $\mathrm{n} / \mathrm{a}$ & $\mathrm{n} / \mathrm{a}$ & Manual & Both & $\mathrm{BA} 17,18,19^{\mathrm{a}}$ & BL60-67 \\
\hline Gareus et al. $[10]^{\mathrm{b}}$ & $8 / 6$ & $60 \mathrm{~s}$ & Other & $\mathrm{n} / \mathrm{a}$ & $P<.05$ & Manual & None & None & GB37 \\
\hline Siedentopf et al. [11] & 10 & $40 \mathrm{~s}$ & FFX & $\begin{array}{c}\text { Cluster } \\
\text { level }\end{array}$ & $P<.05$ & Laser & Activations & BA18, 19 & BL67 \\
\hline Li et al. [12] & 18 & $20 \mathrm{~s}$ & $\mathrm{RFX}^{\mathrm{c}}$ & None & $P<.001$ & $\begin{array}{l}\text { Manual/ } \\
\text { electr. }\end{array}$ & Both & BA17, 18, 19 & $\begin{array}{c}\text { BL60, } 65,66 \\
67\end{array}$ \\
\hline Litscher et al. [13] & 1 & $60 \mathrm{~s}$ & None & $\begin{array}{c}\text { Cluster } \\
\text { level }\end{array}$ & $P<.05$ & Laser & Activations & BA19 & BL67, BL60 \\
\hline Parrish et al. $[14]^{\mathrm{d}}$ & 12 & $300 \mathrm{~s}$ & FFX & None & $\mathrm{n} / \mathrm{a}$ & Manual & Activations & $\mathrm{BA} 17,18,19^{\mathrm{a}}$ & BL60 \\
\hline Kong et al. $[15,16]$ & $6^{e}$ & $30 \mathrm{~s}$ & RFX & None & $P<.001$ & Electrical & Deactivations & BA17, 18, 19 & GB37, BL60 \\
\hline \multicolumn{10}{|l|}{ Hearing-related acupoints } \\
\hline Parrish et al. $[14]^{\mathrm{d}}$ & 12 & $300 \mathrm{~s}$ & FFX & None & $\mathrm{n} / \mathrm{a}$ & Manual & Activations & $\mathrm{BA} 22,41,42^{\mathrm{a}}$ & KID3 \\
\hline Wesolowski et al. [17] ${ }^{\mathrm{b}}$ & 20 & $30 \mathrm{~s}$ & RFX & None & $P<.001$ & Manual & None & None & GB43 \\
\hline
\end{tabular}

${ }^{\mathrm{a}}$ Derived from figures (not reported in article); ${ }^{\mathrm{b}}$ Studies with negative results; ${ }^{\mathrm{c}}$ Only subjects with activations on first level were included in second-level analysis; ${ }^{\mathrm{d}}$ Study covers both visual and auditory points; ${ }^{\mathrm{e}}$ Each subject was measured six times resulting in an effective $n=20$ (according to the authors).

that will, however, not be covered here. While the variability of results is obvious between studies, Kong et al. [15] have also assessed the variability between and within subjects by measuring the test-retest reliability of acupuncture-fMRI. In their interesting approach six subjects underwent the same fMRI scanning procedure six times while receiving acupuncture. They found cortical activations observed under acupuncture stimulation to be much less consistent than those observed under the control task (finger-tapping).

Later in this review, we will give possible explanations for this variability as well as for other divergent results. Our hypothesis is that most of this variability is attributable to methodological problems that can even lead to false positive results.

We will cover a number of basic issues, that should be kept in mind while using fMRI to measure effects of acupuncture, namely how to interpret deactivations, which baseline to choose, how to control attention and why the choice of appropriate group statistics is so important.

\section{Unsolved Problems of Acupuncture-fMRI Studies}

4.1. The Issue of Deactivations. The frequent occurrence of deactivations in the studies analyzed here must be considered carefully. As Table 1 shows, three out of the seven studies using vision-related acupoints report deactivations in some part of the visual cortex. The origin of deactivations in fMRI studies has only recently been clarified [24]. From the mathematical point of view, deactivations are nothing more than negative correlations between the stimulation time course and the Blood Oxygenation Level Dependency (BOLD) signal. Let us assume that these changes in the BOLD signal are related to the stimulus. In this case there exist two possible explanations, namely, an actual reduction of neuronal activity caused by the stimulation (e.g., acupuncture) as compared to the baseline state or by neuronal activity that gets stronger in the baseline condition as compared to stimulation. Unfortunately these two possibilities cannot be distinguished, if one compares directly against baseline. Even if one compares two kinds of stimulation (e.g., real and placebo acupuncture), each of them has been measured directly against baseline. All this can only lead to valid results, if the baseline is well defined and stationary. However, the following section will show that, this is not the usual case.

4.2. Which Baseline to Use? Almost all acupuncture-fMRI studies conducted so far have used "eyes closed" as a baseline for their measurements. That means, subjects were asked to close their eyes and to think of nothing special, while lying in the scanner. In some studies subjects were instructed to continuously focus on the stimulation, however, their actual level of attention was not controlled. The problematic aspect of this "low" baseline is, that the cortical activity associated with it is neither well defined nor stationary $[25,26]$. As known for some time now, brain activity does not cease, when the subject is at rest. On the contrary there are several networks active when subjects are lying inside the scanner "doing nothing" [27, 28]. We will talk about these so called resting state networks (RSN) in more detail later. As Brandt has shown [29], mere closing of the eyes can lead to cortical activations, especially in visual and auditory cortices making its use in experiments on vision and hearing questionable.

4.2.1. Uncontrolled Attention. Another problem when using "eyes closed" as a baseline is that the attention of subjects remains totally uncontrolled. Thus, they will probably focus on the acupuncture stimulation and pursue other cognitive tasks in the time between the stimulation blocks. These changes in attention can lead to cortical activations or 
deactivations as shown by a number of groups. One area that has often been shown to be activated or deactivated in attention changes is (again) the visual cortex [30-32]. Furthermore, a so called "default mode network" (DMN) [33] exists, that is usually active when subjects are doing nothing and thus often gets deactivated, when attention is drawn to some stimulation or cognitive task. This RSN comprises parts of the posterior parietal cortex, occipitoparietal junction, precuneus, posterior cingulate cortex and frontal pole. The idea that attention is responsible for some deactivations observed in acupuncture-fMRI studies is not new and has been put forth by several authors [16, 34]. The study of Kong et al. [16] is a particularly good example since the typical pattern of posterior areas of the DMN can be identified in the paper (Figure 2, top row), where deactivations under electro-acupuncture of a vision-related acupoint are reported. Consequently, the authors of this study consider the possibility that their results are caused by changes in attention rather than by the acupuncture stimulation itself.

Since two of the studies reviewed here $[11,13]$ used laser acupuncture stimulation, we must admit that at least for these studies, uncontrolled changes in attention can hardly explain the results. This is because in laser acupuncture the experimental design is double-blinded and the subject does not feel whether the laser is switched on or off. Thus its attention is not influenced by changing sensory inputs.

4.2.2. Resting State. So far we have assumed that activations observed in acupuncture-fMRI studies were related to the stimulation in some way. However, there is also the opposite possibility of a purely random (anti-)correlation between the stimulation time course and the BOLD signal. As we have seen, the brain is not inactive, when the subject is at rest. On the contrary intrinsic brain activity called resting state is present at all times. This intrinsic activity of the brain at rest has recently drawn the attention of many scientists, especially in the field of fMRI. After PET experiments had shown task- independent deactivations, Raichle proposed the idea of a default mode of brain function $[25,33]$. In recent years, many fMRI studies have been conducted and several additional RSNs have been identified [27, 28]. The frequency range of signal fluctuations in RSNs has been assessed by several groups finding values of $\sim 0-0.1 \mathrm{~Hz}$ [35]. As several authors have pointed out, this frequency range considerably overlaps with repetition frequencies of standard block-design time courses used in fMRI [27, 35]. For example, a block length of $30 \mathrm{~s}$ plus rest blocks of equal length leads to a repetition frequency of $\sim 0.017 \mathrm{~Hz}$. In the case of an incidental phase correlation of intrinsic fluctuations with the time course of an fMRI experiment this can easily result in false positive activations or-in the case of an anti-correlation-to deactivations. One could argue that any cognitive task interrupts the resting state since the brain is no longer at rest. However, several studies have shown, that this is not the case $[36,37]$. The results of Greicius et al. are especially relevant for acupuncture-fMRI, because they show that resting state activity is almost left unchanged in case of a passive, block-design, sensory task (such as manual needle acupuncture). Furthermore, the investigations by the groups of Litscher and Siedentopf were performed using laser needle stimulation, where the subject usually does not even notice if the laser is switched on or off. Because of the purely random character of the phase correlations explained here, two general statements can be made: firstly, using conservative thresholds only few subjects will show these false positive activations. Without further assumptions this effect could in principle explain results on single subject level as reported by the groups of Cho [3] and Litscher [13] but not group results. However, in the section on statistical group analyses we will show that some group statistics can easily turn strong single subject activations into significant group results. The second general statement is that there should be no preference of activations or deactivations, since correlations are as likely to happen as anti-correlations. This is more or less exactly, what we observe in acupuncture-fMRI studies. The amount of activations and deactivations is about the same on group level as well as between and within subject results (where these are reported). For example in the paper by Kong [15, Figures 5 and 6]; they found about the same amount of activations and deactivations. Possible cortical areas for such incidental (de-)activations theoretically include all those that are regularly found in resting state experiments. What is most important for the studies reviewed here is that three out of ten RSN as reported by Beckmann et al. [27] comprise either the visual or the auditory cortex, offering an alternative explanation for the (de-)activations found in these areas in acupuncture-fMRI experiments.

4.3. The Choice of Group Statistic. In fMRI two basic approaches are commonly used for group analyses: Fixedeffects (FFX) and random-effects analyses (RFX). While an RFX takes into account the between-subject-variance of the activations found on single subject level, FFX only considers within-subject-variance. Table 1 reveals that most of the positive results on visual and auditory activations under acupuncture stem from studies that either used FFXs or no group statistics at all.

4.3.1. FFX. It has long been known, that FFX suffers from a high susceptibility to outliers, that is, single subjects with strong activations, as it only accounts for within-subject variance [38]. Hence a single subject with a strong activation can in fact drive a whole FFX to produce significant results at group level that look as if the majority of subjects had shown this activation. This effect is especially relevant in the case of small sample sizes. As shown above there exists a mechanism that can produce such strong activations in single subjects, namely incidental phase correlations of resting state activity and the time course of the block-design. In fact, the use of FFX is deprecated today by most scientists exactly for this reason. A recent study by Smith et al. has quantified the effect of intrinsic brain activity on fMRI time series in single subject and FFX and has shown to be quite substantial [39].

4.3.2. RFX. Only three out of nine studies covered in this review have used RFX and even these reported their results on an uncorrected level, that is, without applying any of 
the common correction methods for multiple comparisons. In fact, these are the only studies of those reviewed here that applied state-of-the-art statistics. A closer look at one of these RFX studies [12] reveals that only subjects showing visual activations on single subject level were included in the group analysis. This approach will almost certainly lead to positive group results. The two remaining RFX studies either yielded null results [17] or results that were interpreted by the authors as resulting from changes in attention [16].

\section{Synopsis and Possible Solutions}

While we are far from questioning the validity of using fMRI for the study of acupuncture effects, we think that visual and auditory activations in the studies reviewed here were probably not a direct result of acupuncture stimulation. We have seen that there exist several mechanisms other than acupuncture that can explain such activations. Using "eyes closed" as a baseline leaves attention of the subjects uncontrolled and can therefore lead to (de-)activations due to changes in attention as a result of needle stimulation. Mere closing of the eyes can also lead to activations in visual and auditory cortices. Furthermore, acupuncture may be too weak a stimulus to suppress resting state activity of the brain which may on its part lead to false positive (de-)activations in case of incidental phase correlations with the stimulus time course. RSN comprise both visual and auditory cortical areas.

Most of these problems can be overcome by using a different better controlled baseline. One possibility is a continuous attention task throughout the entire experiment. Attention may be controlled for, for example, by a changing visual stimulus, where the subject has to react to these changes by pressing a button. Although this is an untypical situation as compared to a standard therapeutic acupuncture treatment, we think the advantages clearly outweigh the disadvantages here. If the implementation of an attention task is impossible for any reason, researchers should at least familiarize themselves with the typical patterns of resting state activity.

As we have seen, the formerly widely used fixed-effect group analysis can easily turn strong activations of single subjects into positive group results even if the rest of the group did not show these activations. As a result this statistical method should not be used any more. Instead RFX should be the method of choice. These require a minimum of about 12 subjects; however, the aim should be to report results with a corrected threshold, which may require double the amount of subjects [40].

A final point to question the specificity of visual and auditory activations under acupuncture stimulation is that Parrish found hearing-related acupoints to activate visual cortical areas as well (see [14, Figures 1 and 2]). Furthermore, a large number of studies that did not use vision-related acupoints nevertheless found activations in visual cortical areas $[9,34,41-53]$.

After pointing out the most important methodological problems of past acupuncture-fMRI studies, and showing their possible impact on study results, we would like to emphasize that our results do not contradict the existence of possible therapeutic effects of acupuncture. To name just one result, Litscher and colleagues have shown that laser needle stimulation of vision-related acupoints can increase blood flow in the ophthalmic artery, which is the largest supplying vessel of the eye [54]. This constitutes an alternative explanation for possible therapeutic effects of these acupoints. We hope that adopting the solutions offered here will lead to more appropriate studies and finally enable us to unravel the underlying mechanisms of acupuncture.

\section{Funding}

F.B. would like to thank Manfred Köhnlechner Stiftung for funding support. C.H. was supported by BMBF (German Ministry of Education and Science).

\section{Acknowledgment}

The authors would like to thank Simon Baudrexel for helpful discussions.

\section{References}

[1] T. Yoshida, C. Tanaka, M. Umeda, T. Higuchi, M. Fukunaga, and S. Naruse, "Non-invasive measurement of brain activity using functional MRI: toward the study of brain response to acupuncture stimulation," The American Journal of Chinese Medicine, vol. 23, no. 3-4, pp. 319-325, 1995.

[2] K. K. S. Hui, J. Liu, and K. K. Kwong, "Functional mapping of the human brain during acupuncture with magnetic resonance imaging somatosensory cortex activation," World Journal of Acupuncture-Moxibustion, vol. 7, pp. 44-49, 1997.

[3] Z. H. Cho, S. C. Chung, J. P. Jones, J. B. Park, H. J. Park, H. J. Lee et al., "New findings of the correlation between acupoints and corresponding brain cortices using functional MRI," Proceedings of the National Academy of Sciences of the United States of America, vol. 95, pp. 2670-2673, 1998.

[4] Z. H. Cho, S. C. Chung, H. J. Lee, E. K. Wong, and B. I. Min, "Retraction. New findings of the correlation between acupoints and corresponding brain cortices using functional MRI," Proceedings of the National Academy of Sciences of the United States of America, vol. 103, p. 10527, 2006.

[5] G. T. Lewith, P. J. White, and J. Pariente, "Investigating acupuncture using brain imaging techniques: the current state of play," Evidence-Based Complementary and Alternative Medicine, vol. 2, no. 3, pp. 315-319, 2005.

[6] G. Litscher, "Bioengineering assessment of acupuncture, part 4: functional magnetic resonance imaging," Critical Reviews in Biomedical Engineering, vol. 34, no. 4, pp. 327-345, 2006.

[7] R. P. Dhond, N. Kettner, and V. Napadow, "Neuroimaging acupuncture effects in the human brain," Journal of Alternative and Complementary Medicine, vol. 13, no. 6, pp. 603-616, 2007.

[8] A. R. White, J. Filshie, and T. M. Cummings, "Clinical trials of acupuncture: consensus recommendations for optimal treatment, sham controls and blinding," Complementary Therapies in Medicine, vol. 9, no. 4, pp. 237-245, 2001.

[9] J. Kong, L. Ma, R. L. Gollub, J. Wei, X. Yang, D. Li et al., "A pilot study of functional magnetic resonance imaging of 
the brain during manual and electro acupuncture stimulation of acupuncture point (LI-4 Hegu) in normal subjects reveals differential brain activation between methods," Journal of Alternative and Complementary Medicine, vol. 8, pp. 411-419, 2002.

[10] I. K. Gareus, M. Lacour, A.-C. Schulte, and J. Hennig, "Is there a BOLD response of the visual cortex on stimulation of the vision-related acupoint GB 37?" Journal of Magnetic Resonance Imaging, vol. 15, no. 3, pp. 227-232, 2002.

[11] C. M. Siedentopf, S. M. Golaszewski, F. M. Mottaghy, C. C. Ruff, S. Felber, and A. Schlager, "Functional magnetic resonance imaging detects activation of the visual association cortex during laser acupuncture of the foot in humans," Neuroscience Letters, vol. 327, no. 1, pp. 53-56, 2002.

[12] G. Li, R. T. F. Cheung, Q.-Y. Ma, and E. S. Yang, "Visual cortical activations on fMRI upon stimulation of the vision-implicated acupoints," NeuroReport, vol. 14, no. 5, pp. 669-673, 2003.

[13] G. Litscher, D. Rachbauer, S. Ropele, L. Wang, D. Schikora, F. Fazekas et al., "Acupuncture using laser needles modulates brain function: first evidence from functional transcranial Doppler sonography and functional magnetic resonance imaging," Lasers in Medical Science, vol. 19, pp. 6-11, 2004.

[14] T. B. Parrish, A. Schaeffer, M. Catanese, and M. J. Rogel, "Functional magnetic resonance imaging of real and sham acupuncture. Noninvasively measuring cortical activation from acupuncture," IEEE Engineering in Medicine and Biology Magazine, vol. 24, pp. 35-40, 2005.

[15] J. Kong, R. L. Gollub, J. M. Webb, J.-T. Kong, M. G. Vangel, and K. Kwong, "Test-retest study of fMRI signal change evoked by electroacupuncture stimulation," NeuroImage, vol. 34, no. 3, pp. 1171-1181, 2007.

[16] J. Kong, T. J. Kaptchuk, J. M. Webb et al., "Functional neuroanatomical investigation of vision-related acupuncture point specificity-a multisession fMRI study," Human Brain Mapping, vol. 30, no. 1, pp. 38-46, 2009.

[17] T. Wesolowski, M. Lotze, M. Domin et al., "Acupuncture reveals no specific effect on primary auditory cortex: a functional magnetic resonance imaging study," NeuroReport, vol. 20, no. 2, pp. 116-120, 2009.

[18] A. Amedi, G. Jacobson, T. Hendler, R. Malach, and E. Zohary, "Convergence of visual and tactile shape processing in the human lateral occipital complex zohary," Cerebral Cortex, vol. 12, no. 11, pp. 1202-1212, 2002.

[19] C. Kayser, C. I. Petkov, M. Augath, and N. K. Logothetis, "Integration of touch and sound in auditory cortex," Neuron, vol. 48, no. 2, pp. 373-384, 2005.

[20] M. Schürmann, G. Caetano, Y. Hlushchuk, V. Jousmäki, and R. Hari, "Touch activates human auditory cortex," NeuroImage, vol. 30, no. 4, pp. 1325-1331, 2006.

[21] P. Deadman, K. Baker, and M. Al-Khafaji, A Manual of Acupuncture.Journal of Chinese Medicine Publications, sussex, Hove, UK, 1998.

[22] Z. Cho, C. Na, E. Wong, S. Lee, and I. Hong, "Investigation of acupunctureusing brain functional magnetic resonance imaging," in Computer Controlled Acupuncture, G. Litscher and Z. Cho, Eds., pp. 45-64, Pabst Scientific, Lengerich, Germany, 2000.

[23] Z. Cho, C. Na, E. Wong, S. Lee, and I. Hong, "Functional magnetic resonance imaging of the brain in the investigation of acupuncture," in Clinical Acupuncture-Scientific Basis, G. Stux and R. Hammerschlag, Eds., pp. 85-90, Springer, Berlin, Germany, 2001.

[24] R. G. Shulman, D. L. Rothman, and F. Hyder, "A BOLD search for baseline," NeuroImage, vol. 36, no. 2, pp. 277-281, 2007.
[25] D. A. Gusnard and M. E. Raichle, "Searching for a baseline: functional imaging and the resting human brain," Nature Reviews Neuroscience, vol. 2, no. 10, pp. 685-694, 2001.

[26] C. E. L. Stark and L. R. Squire, "When zero is not zero: the problem of ambiguous baseline conditions in fMRI," Proceedings of the National Academy of Sciences of the United States of America, vol. 98, no. 22, pp. 12760-12765, 2001.

[27] C. F. Beckmann, M. DeLuca, J. T. Devlin, and S. M. Smith, "Investigations into resting-state connectivity using independent component analysis," Philosophical Transactions of the Royal Society B: Biological Sciences, vol. 360, no. 1457, pp. 1001-1013, 2005.

[28] M. de Luca, C. F. Beckmann, N. de Stefano, P. M. Matthews, and S. M. Smith, "fMRI resting state networks define distinct modes of long-distance interactions in the human brain," NeuroImage, vol. 29, no. 4, pp. 1359-1367, 2006.

[29] T. H. Brandt, "How to see what you are looking for in fMRI and PET_-or the crucial baseline condition," Journal of Neurology, vol. 253, no. 5, pp. 551-555, 2006.

[30] S. Kastner, M. A. Pinsk, P. De Weerd, R. Desimone, and L. G. Ungerleider, "Increased activity in human visual cortex during directed attention in the absence of visual stimulation," Neuron, vol. 22, no. 4, pp. 751-761, 1999.

[31] L. Pessoa, S. Kastner, and L. G. Ungerleider, "Neuroimaging studies of attention: from modulation of sensory processing to top-down control," Journal of Neuroscience, vol. 23, no. 10, pp. 3990-3998, 2003.

[32] W. D. Hairston, D. A. Hodges, R. Casanova et al., "Closing the mind's eye: deactivation of visual cortex related to auditory task difficulty," NeuroReport, vol. 19, no. 2, pp. 151-154, 2008.

[33] M. E. Raichle, A. M. MacLeod, A. Z. Snyder, W. J. Powers, D. A. Gusnard, and G. L. Shulman, "A default mode of brain function," Proceedings of the National Academy of Sciences of the United States of America, vol. 98, no. 2, pp. 676-682, 2001.

[34] K. K. S. Hui, J. Liu, O. Marina et al., "The integrated response of the human cerebro-cerebellar and limbic systems to acupuncture stimulation at ST 36 as evidenced by fMRI," NeuroImage, vol. 27, no. 3, pp. 479-496, 2005.

[35] D. Cordes, V. M. Haughton, K. Arfanakis et al., "Frequencies contributing to functional connectivity in the cerebral cortex in "resting-state" data," American Journal of Neuroradiology, vol. 22, no. 7, pp. 1326-1333, 2001.

[36] M. D. Greicius and V. Menon, "Default-mode activity during a passive sensory task: uncoupled from deactivation but impacting activation," Journal of Cognitive Neuroscience, vol. 16, no. 9, pp. 1484-1492, 2004.

[37] P. Fransson, "How default is the default mode of brain function?. Further evidence from intrinsic BOLD signal fluctuations," Neuropsychologia, vol. 44, no. 14, pp. 2836-2845, 2006.

[38] K. J. Friston, A. P. Holmes, and K. J. Worsley, "How many subjects constitute a study?" NeuroImage, vol. 10, no. 1, pp. $1-5,1999$.

[39] A. T. Smith, K. D. Singh, and J. H. Balsters, "A comment on the severity of the effects of non-white noise in fMRI time-series," NeuroImage, vol. 36, no. 2, pp. 282-288, 2007.

[40] J. E. Desmond and G. H. Glover, "Estimating sample size in functional MRI (fMRI) neuroimaging studies: statistical power analyses," Journal of Neuroscience Methods, vol. 118, no. 2, pp. 115-128, 2002.

[41] M.-T. Wu, J.-M. Sheen, K.-H. Chuang et al., "Neuronal specificity of acupuncture response: a fMRI study with electroacupuncture," NeuroImage, vol. 16, no. 4, pp. 10281037, 2002. 
[42] G. Li, H.-L. Liu, R. T. F. Cheung et al., "An fMRI study comparing brain activation between word generation and electrical stimulation of language-implicated acupoints," Human Brain Mapping, vol. 18, no. 3, pp. 233-238, 2003.

[43] B. Yan, K. Li, J. Xu et al., "Acupoint-specific fMRI patterns in human brain," Neuroscience Letters, vol. 383, no. 3, pp. 236240, 2005.

[44] K. Li, B. Shan, J. Xu et al., "Changes in fMRI in the human brain related to different durations of manual acupuncture needling," Journal of Alternative and Complementary Medicine, vol. 12, no. 7, pp. 615-623, 2006.

[45] Y. Y. Xiao, X. K. Chen, L. Du, R. Q. Pei, F. Y. Chen, G. R. Liu et al., "The brain mapping on reinforcement acupuncture stimulation at ST36 (zusanli) evidenced by fMRI," Conference Proceedings-IEEE Engineering in Medicine and Biology Society, vol. 1, pp. 1036-1039, 2006.

[46] W. Wang, L. Liu, X. Zhi, J. Huang, D. Liu, H. Wang et al., "Study on the regulatory effect of electro-acupuncture on hegu point (LI4) in cerebral response with functional magnetic resonance imaging," Chinese Journal of Integrative Medicine, vol. 13, pp. 10-16, 2007.

[47] S.-M. Wang, R. T. Constable, F. S. Tokoglu, D. A. Weiss, D. Freyle, and Z. N. Kain, "Acupuncture-induced blood oxygenation level-dependent signals in awake and anesthetized volunteers: a pilot study," Anesthesia and Analgesia, vol. 105, no. 2, pp. 499-506, 2007.

[48] J. Zhang, X. Cao, J. Lie, W. Tang, H. Liu, and X. Fenga, "Neuronal specificity of needling acupoints at same meridian: a control functional magnetic resonance imaging study with electroacupuncture," Acupuncture Electro-Therapeutics Research, vol. 32, pp. 179-193, 2007.

[49] R. P. Dhond, C. Yeh, K. Park, N. Kettner, and V. Napadow, "Acupuncture modulates resting state connectivity in default and sensorimotor brain networks," Pain, vol. 136, no. 3, pp. 407-418, 2008.

[50] H. MacPherson, G. Green, A. Nevado et al., "Brain imaging of acupuncture: comparing superficial with deep needling," Neuroscience Letters, vol. 434, no. 1, pp. 144-149, 2008.

[51] L . Li, H. Liu, Y. Li, J. Xu, B. Shan, D Gong et al., "The human brain response to acupuncture on same-meridian acupoints: evidence from an fMRI study," Journal of Alternative and Complementary Medicine, vol. 14, pp. 673-678, 2008.

[52] Y. Wu, Z. Jin, K. Li et al., "Effect of acupuncture on the brain in children with spastic cerebral palsy using functional neuroimaging (fMRI)," Journal of Child Neurology, vol. 23, no. 11, pp. 1267-1274, 2008.

[53] Y. Zhou and J. Jin, "Effect of acupuncture given at the HT 7, ST 36, ST 40 and KI 3 acupoints on various parts of the brains of Alzheimer's disease patients," Acupuncture and Electro-Therapeutics Research, vol. 33, no. 1-2, pp. 9-17, 2008.

[54] G. Litscher and D. Schikora, "Cerebral vascular effects of noninvasive laserneedles measured by transorbital and transtemporal Doppler sonography," Lasers in Medical Science, vol. 17, no. 4, pp. 289-295, 2002. 


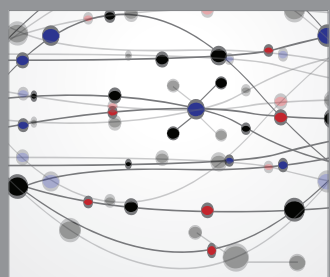

The Scientific World Journal
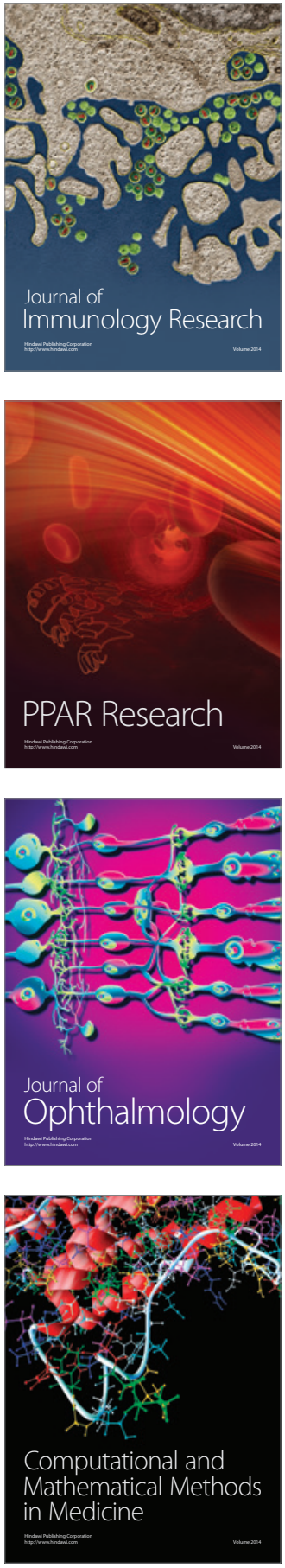

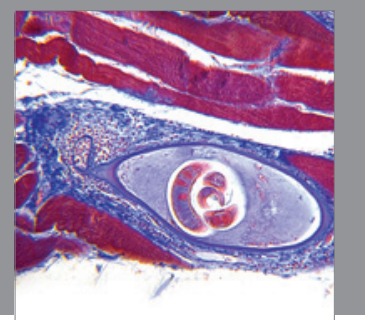

Gastroenterology

Research and Practice
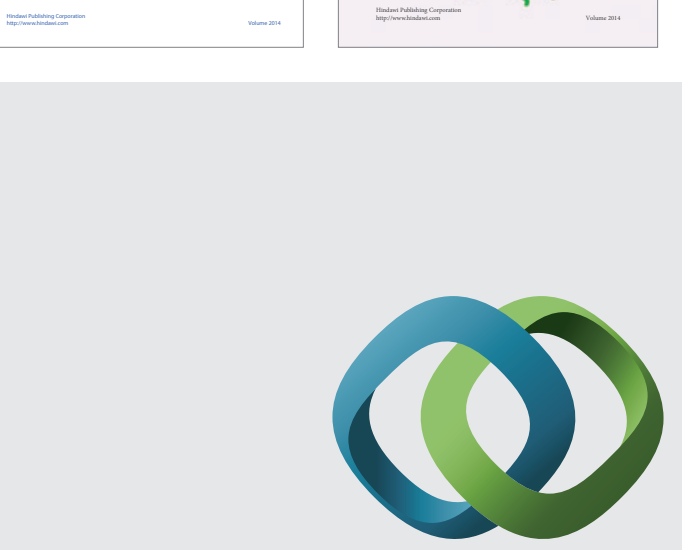

\section{Hindawi}

Submit your manuscripts at

http://www.hindawi.com
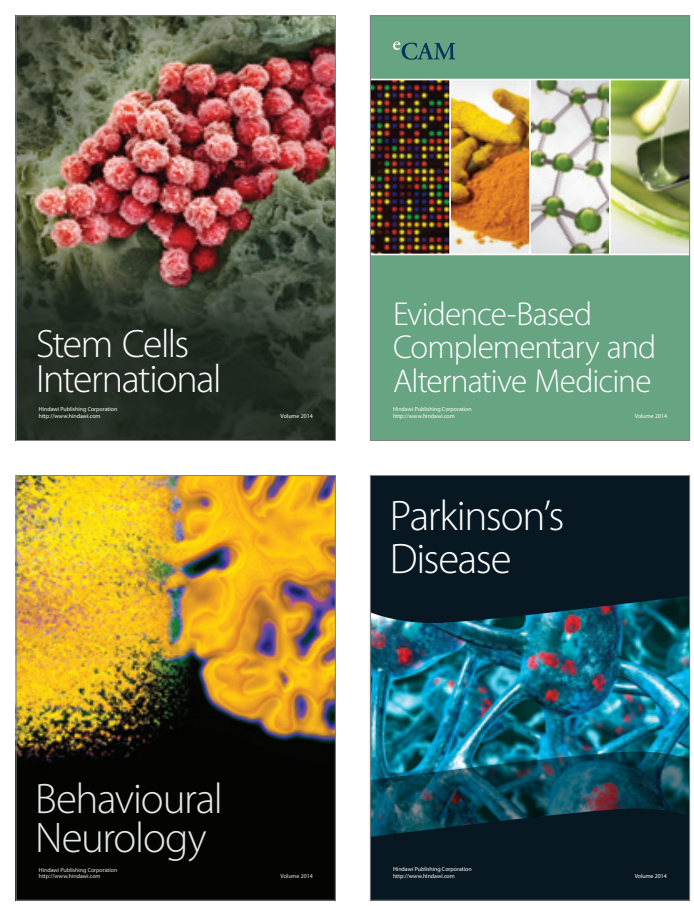

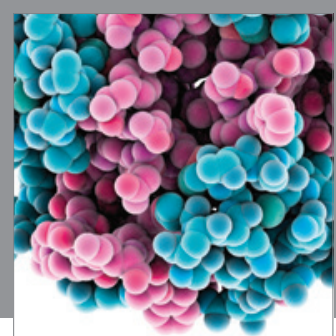

Journal of
Diabetes Research

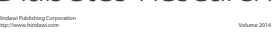

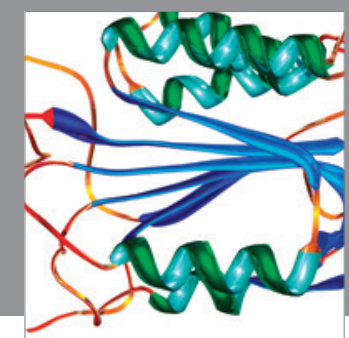

Disease Markers
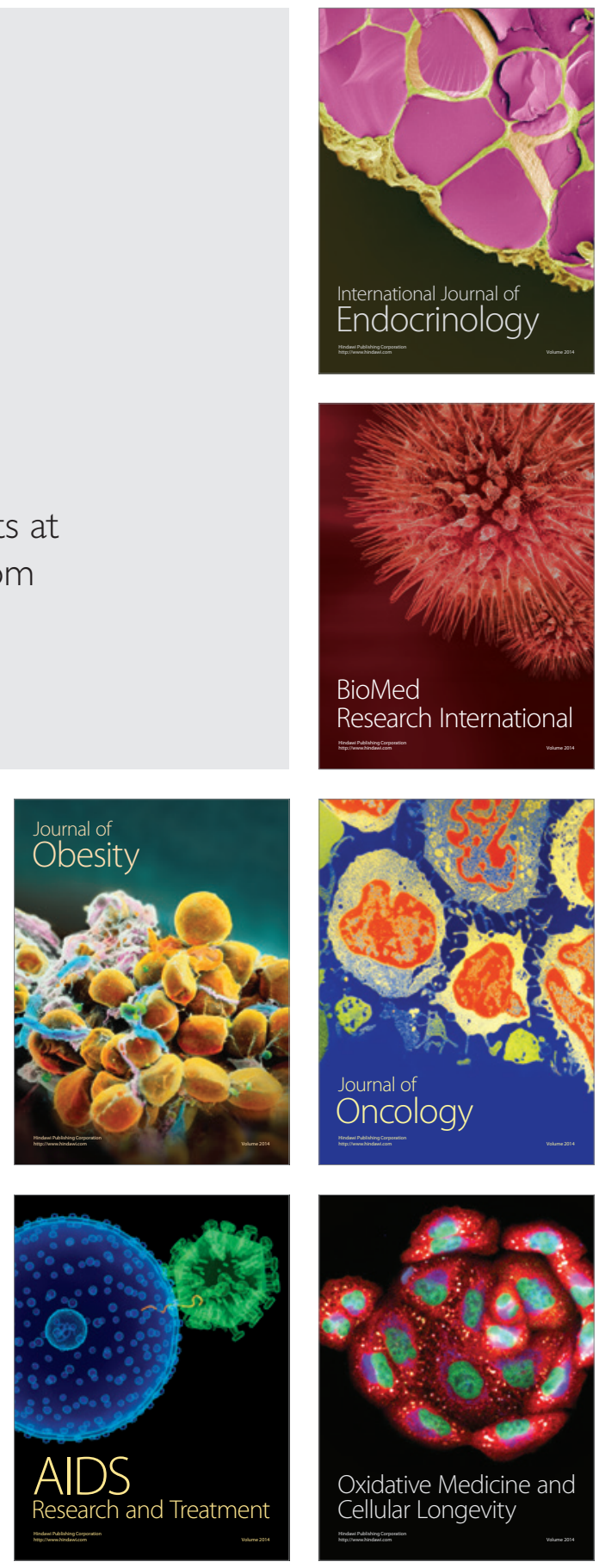